\title{
The effect of miR-496 on elF3h in lung invasive adenocarcinoma
}

\author{
Ke Zhang and Ji-Chun Tong ${ }^{凶}$ \\ Department of Cardiothoracic Surgery, Changzhou No. 2 People's Hospital, The Affiliated Hospital of Nanjing Medical University, Changzhou, \\ Jiangsu 213000, P.R. China
}

This study explores the correlation between microRNA-496 (miR-496) expression and eukaryotic translation initiation factor $3 \mathrm{H}$ (elF3h) expression in lung invasive adenocarcinoma. A total of 71 patients with lung adenocarcinoma were included in the study. The tumor tissue samples were collected together with the normal lung tissue samples that were acquired more than $2 \mathrm{~cm}$ away from the edge of the tumor). Expression of miR-496 was detected using real-time fluorescence quantitative PCR. Expression of $\mathrm{Ki} 67$ and $\mathrm{Bcl}-2$ in lung adenocarcinoma was detected with Western blotting, and expression of elF3hwas detected with immunohistochemistry. Expression of miR-496 was significantly lower in the tumor than in normal lung tissue $(P<0.05)$. miR-496 expression was significantly decreased in the tumor samples from the patients with large tumor maximum diameter, pleural recidivism, lymph node metastasis, and TNM stage III + IV, compared to the samples from the patients with small tumor diameter, no pleural recidivism, no lymph node metastasis, and TNM stage I + II $(P<0.05)$. No significant difference in miR-496 expression was identified among the groups of different sex, age, and tumor differentiation degree $(P>0.05)$. Low expression of miR-496 correlated with a shorter survival time of the patients $(P<0.05)$. Moreover, miR-496 expression was negatively correlated with $\mathrm{Ki} 67, \mathrm{Bcl}-2$ and elF3h expression. We speculate that and miR-496 has the potential to regulates elF $3 \mathrm{~h}$ expression, and low expression of miR-496 in lung adenocarcinoma may be related to the occurrence and development of the tumor. Mir-496 may be an independent prognostic factor in lung adenocarcinoma.

Keywords: lung adenocarcinoma, miR-496, elF3h, proliferation, apoptosis, prognosis

Received: 17 September, 2020; revised: 29 October, 2020; accepted: 30 October, 2020; available on-line: 26 May, 2021

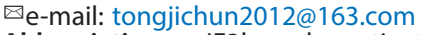

Abbreviations: elF3h, eukaryotic translation initiation factor $3 h$; miRNA, micro-RNA; NSCLC, Non-small cell lung cancer

\section{INTRODUCTION}

Lung cancer is one of the malignant tumors with the highest morbidity and mortality. Non-small cell lung cancer (NSCLC), which accounts for about $80 \%$ of all lung cancers, can be further divided into subtypes including lung adenocarcinoma, lung squamous cell carcinoma, and lung large cell carcinoma. Among them, lung adenocarcinoma is the most common subtype of all lung cancers, accounting for about $40 \%$ of all lung cancers and show- ing an increasing trend in recent years (Meng et al., 2013; Wu et al., 2017).

miRNA is a class of 18-25 nucleotide non-coding single-stranded RNA that is common in eukaryotes. Recently, the role of miRNA in promoting the proliferation and metastasis as well as regulating the apoptosis of tumor cells has been found by researchers (Mi et al., 2017), and the abnormal expression of micro-RNA (miRNA) in tumor progression gathered attention. Mir-496 is located on human chromosome 14, and its biological function is not clear. Some studies have shown that low expression of miR-496 correlates with aging and plays an important role in the formation and differentiation of bone cells (Palmieri et al., 2010). It was found that overexpression of miR-496 could inhibit the proliferation of breast cancer cells and was MBD2 dependent (Alvarado, 2013). At present, there are few reports about miR-496, and its biological function in tumor development is not clear. Up to now, there is no report about miR-496 expression in lung adenocarcinoma.

Eukaryotic translation initiation factor 3 subunit $\mathrm{H}$ (eIF3h) is a translation initiation complex containing 1013 different subunits, which can stably bind Met complex to $40 \mathrm{~S}$ ribosome subunit, cause cell dysplasia and induce tumor progression (Choudhuri et al., 2013). Savianinen and others (Savinainen, et al., 2004) found that eIF3h expression was related to the stage of prostate cancer. Zhang and others (Zhang et al., 2007) found that overexpression of eIF3h in NIH3T3 cells can lead to malignant transformation of the cells. It has been shown that reducing eIF3h expression level with interfering RNA in breast cancer and prostate cancer cell lines can slow down the proliferation of these cells (Zhang, 2008).

In this study, we screened the TCGA database for differentially expressed miRNA and selected miR-496, which is most related to lung adenocarcinoma and rarely reported in the clinical studies. Using TargetScan database, we predicted that eIF3h may be the downstream target gene of miR-496. We explored the expression of miR-496 in postoperative tissues of lung adenocarcinoma and further analyzed the relationship between miR-496 and the patients' clinical features, and prognosis, as well as its correlation with proliferation and apoptosis markers. In addition, we also examined the possible correlation between miR-496 and eIF3h expression, in order to provide theoretical basis for future cancer research.

\section{MATERIALS AND METHODS}

\section{Clinical data}

The patients included in this study were diagnosed with lung adenocarcinoma between September 2013 
and August 2014, based on the following criteria: (1) they were diagnosed in line with the WHO diagnostic criteria of lung adenocarcinoma; (2) they were firstly diagnosed without earlier intervention. Exclusion criteria were as follows: (1) malignant tumors of other organs; (2) receiving preoperative radiotherapy and chemotherapy; (3) controversial diagnosis. A total of 71 cases were observed, including 30 men and 41 women, aged 32 to 85 years (mean 59.3 years). In this group, there were 41 cases of pleural involvement and 30 cases of no pleural involvement, and 22 cases with lymph node metastasis and 49 cases without lymph node metastasis. TNM staging included 10 cases in stage I, 20 cases in stage II, 26 cases in stage III and 15 cases in stage IV. The collected specimens consisted of the tumor tissue (later on referred to as the tumor tissue group) and the normal lung tissue, which was collected $2 \mathrm{~cm}$ away from the margin of the tumor (later on referred to as the normal tissues group). The study was approved by the Ethics Committee of Changzhou No.2 People's Hospital, in line with the basic principles of the Helsinki Declaration with the signed informed consent from all patients.

\section{METHODS}

\section{Total RNA isolation and real-time fluorescence quantitative PCR}

The expression of miR-496 in lung adenocarcinoma and normal lung tissues was detected using real-time fluorescence quantitative PCR. Total RNA was extracted from fresh specimens. The primers were synthesized by Shanghai Jima Technology Co., Ltd., and the reagent was purchased from Invitrogen Company of the United States. The miR-496 upstream primer was 5'-AAATATGCGTGTGATGATGATCGAAA-3'; and the downstream primer was 5'-TCAAAAAAGCCCGGCTACCT-3'. U6 was used as a reference gene. The U6 upstream primer was 5'-GGAACGAGAAAGATTAGC-3', and downstream primer was 5'-TGGAATTCACGAATTCCG-3'. The experimental operation was carried out strictly according to the protocols. The procedure was as follows: $95^{\circ} \mathrm{C} 8$ minutes, 1 cycle; $95^{\circ} \mathrm{C} 25$ seconds, $64^{\circ} \mathrm{C} 20$ seconds, $72^{\circ} \mathrm{C} 20$ seconds, 10 cycles; $93^{\circ} \mathrm{C} 25$ seconds, $60^{\circ} \mathrm{C} 35$ seconds, $72^{\circ} \mathrm{C} 20$ seconds, 35 cycles. The signal was collected at $60^{\circ} \mathrm{C}$, the $\mathrm{Ct}$ value was calculated automatically, and the result was represented by $2^{-\Delta \Delta \mathrm{Ct}} . \Delta \Delta \mathrm{Ct}=[\mathrm{Ct}$ target gene (sample)-Ct GAPDH (sample)]-[Ct target gene (calibration sample)-Ct GAPDH (calibration sample)].

\section{Western blotting}

The expression of $\mathrm{Ki} 67$ and Bcl-2 in lung adenocarcinoma was detected using Western blotting. The experimental operation was carried out strictly according to the protocols. Fresh specimens were used for the protein sample preparation. The reference gene was GAPDH. The electrophoresis gel was applied into the box and exposed to light for $3 \mathrm{~min}$ and developed for 20 seconds. The target protein signal was normalized to GAPDH and analyzed by Image J software.

\section{Immunohistochemistry}

eIF3h protein levels in paraffin-embedded tissues of lung adenocarcinoma were detected with immunohistochemistry using two-step method. Both eIF3h anti- body and DAB were purchased from Beijing Zhongshan Jinqiao Biotechnology Co., Ltd. The concentration of eIF3h antibody was 1:500. Three 400 fold visual fields were selected to calculate the positive rate and the average value were measured.

\section{Statistical analysis}

Data were analyzed with $t$-test, paired $t$-test, or chisquare test using SAS6.12 software. Pearson correlation analysis and $\mathrm{K} / \mathrm{M}$ survival analysis were performed with $P<0.05$ as the test standard.

\section{RESULTS}

\section{miR-496 expression is in lung adenocarcinoma is decreased}

71 Lung adenocarcinoma tissues and 71 normal tissues were examined. The expression of miR-496 in lung adenocarcinoma was significantly lower than in normal tissues $(P<0.05)$ (Fig. 1). The expression of miR-496 in normal tissues group was about 1.37 times higher than in tumor tissues (Table 1).

\section{miR-496 expression correlates with some clinicopathological features}

The miR-496 expression in different clinical case characteristic groups is shown in Table 2. The expression of miR-496 in tumors of maximum diameter $\geq 3$ was significantly lower than in smaller tumors (maximum diameter $<3 \mathrm{~cm})(P<0.05)$. The expression of miR-496 in tumor tissue from the patients with pleural involvement was significantly lower than in the tumors from the patients without pleural involvement $(P<0.05)$. Also, the expression of miR-496 in tumor tissue from the patients with lymph node metastasis was significantly lower than in the tumors from the patients without lymph node metastasis $(P<0.05)$. Likewise, miR-496 expression was significantly lower in TNM stage III-IV than in TNM stage I-II $(P<0.05)$. Gender and age of the patients as well as the degree

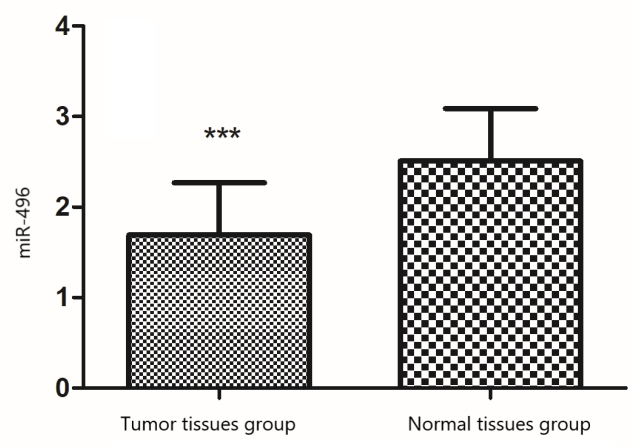

Figure 1. Comparison of miR-496 expression in two groups.

Table 1. Comparison of miR-496 expression in two groups

\begin{tabular}{lllll}
\hline Groups & N of Cases & miR-496 & $t$ & $P$ \\
\hline Observation group & 71 & $1.69 \pm 0.42$ & 6.75 & \\
\hdashline Control group & 71 & $2.31 \pm 0.29$ & & 0.007 \\
\hline
\end{tabular}


Table 2. Comparison of miR-496 expression in groups with different clinicopathological features

\begin{tabular}{|c|c|c|c|c|}
\hline Clinical features & $\mathrm{N}$ of cases & miR-496 & $\mathrm{t}$ & $\mathrm{P}$ \\
\hline \multicolumn{3}{|l|}{ Sex } & \multirow{3}{*}{0.70} & \multirow{3}{*}{0.598} \\
\hline Male & 30 & $1.72 \pm 0.35$ & & \\
\hline Female & 41 & $1.67 \pm 0.37$ & & \\
\hline \multicolumn{3}{|l|}{ Age } & \multirow{3}{*}{0.88} & \multirow{3}{*}{0.442} \\
\hline$<60 y$ & 32 & $1.73 \pm 0.38$ & & \\
\hline$\geq 60 y$ & 39 & $1.66 \pm 0.37$ & & \\
\hline \multicolumn{3}{|c|}{ Differentiation degree } & \multirow{3}{*}{0.95} & \multirow{3}{*}{0.452} \\
\hline Medium-High & 51 & $1.66 \pm 0.45$ & & \\
\hline Low & 20 & $1.74 \pm 0.33$ & & \\
\hline \multicolumn{3}{|c|}{ Tumor maximum diameter } & \multirow{3}{*}{4.96} & \multirow{3}{*}{0.012} \\
\hline$<3 \mathrm{~cm}$ & 31 & $1.85 \pm 0.45$ & & \\
\hline$\geq 3 \mathrm{~cm}$ & 40 & $1.58 \pm 0.35$ & & \\
\hline \multicolumn{3}{|c|}{ Pleural involvement } & \multirow{3}{*}{4.97} & \multirow{3}{*}{0.011} \\
\hline No & 30 & $1.85 \pm 0.37$ & & \\
\hline Yes & 41 & $1.57 \pm 0.36$ & & \\
\hline \multicolumn{3}{|c|}{ Lymph node metastasis } & \multirow{3}{*}{4.98} & \multirow{3}{*}{0.01} \\
\hline No & 49 & $1.79 \pm 0.38$ & & \\
\hline Yes & 22 & $1.51 \pm 0.37$ & & \\
\hline \multicolumn{3}{|l|}{ TNM stages } & \multirow{3}{*}{4.05} & \multirow{3}{*}{0.023} \\
\hline I-II stage & 30 & $1.82 \pm 0.38$ & & \\
\hline III-IV stage & 41 & $1.60 \pm 0.34$ & & \\
\hline
\end{tabular}

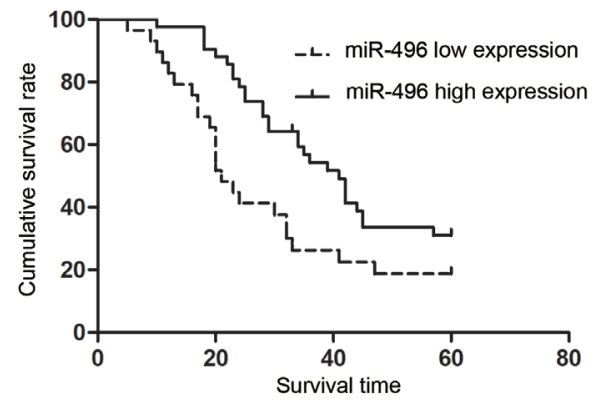

Figure 2. Correlation between miR-496 expression and survival time.

of cell differentiation within the tumor did not have significant correlation with miR-496 expression in the tumor tissue. $(P>0.05)$.

\section{High miR-496 expression correlates with longer survival}

All the patients included in this study were followed for 5 years ( 60 months). The survival time of the patients was between 5 and 60 months, and the median survival time was 20 months. At the end of the 5-year follow-up, 16 patients survived, 52 died, and we lost track of 3. Kaplan-Meier survival plot shown in Fig. 2 depicts the correlation between miR-496 expression and the survival time of the patients. The prognosis was significantly better for the patients with high expression of miR-496 than for the group with low miR-496 expression $\left(\mathrm{X}^{2}=6.55, P=0.002\right)$.

\section{Correlation between miR-496 expression and Ki67and} $\mathrm{BCl}-2$ protein levels is negative in the tumor

The $\mathrm{Ki67}$ and $\mathrm{Bcl}-2$ protein levels in the protein lysates from the tumors were quantified densitometrically and normalized to b-actin. The range of $\mathrm{Ki} 67$ relative protein levels in the tumor was $0.9 / 3.3$, with an average of 2.5 (Fig. 3A). The correlation analysis showed a negative correlation between miR-496 expression and Ki67 relative protein level $(\mathrm{r}=0.50$, $P<0.022$ ) (Fig. 3B). The range of $\mathrm{Bcl}-2$ relative protein levels was 0.5 to 3.8, with an average of 2.4 (Fig. 3A). Based on correlation analysis, a negative correlation was observed between miR-496 expression and Bcl-2 relative protein level $(\mathrm{r}=0.49, P<0.037)$ (Fig. 3B).

\section{Correlation between miR-496 expression and elF3h protein level in the tumor}

The eIF3h staining in lung adenocarcinoma tissue is shown in Fig. 4. The eIF3h expression was observed in cytoplasm and/or cell membrane. The percentage of eIF3h positive cells in the tumor tissue was between $8 \%$ and $80 \%$, with an average of $39.5 \%$. The expression of miR-496 was negatively correlated with percentage of eIF3h positive cells $(\mathrm{r}=0.5, P<0.011)$ (Fig. 5).

\section{DISCUSSION}

The etiology of lung adenocarcinoma is believed to depend on a variety of molecular biological pathways. The abnormal expression of miRNAs may pro- 
A

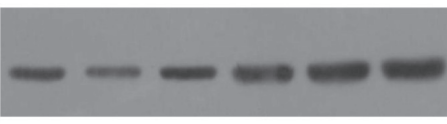

\section{Ki67}

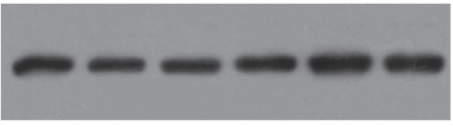

\section{$\mathrm{Bcl}-2$}

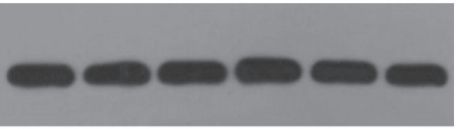

\section{$\beta$-Actin}
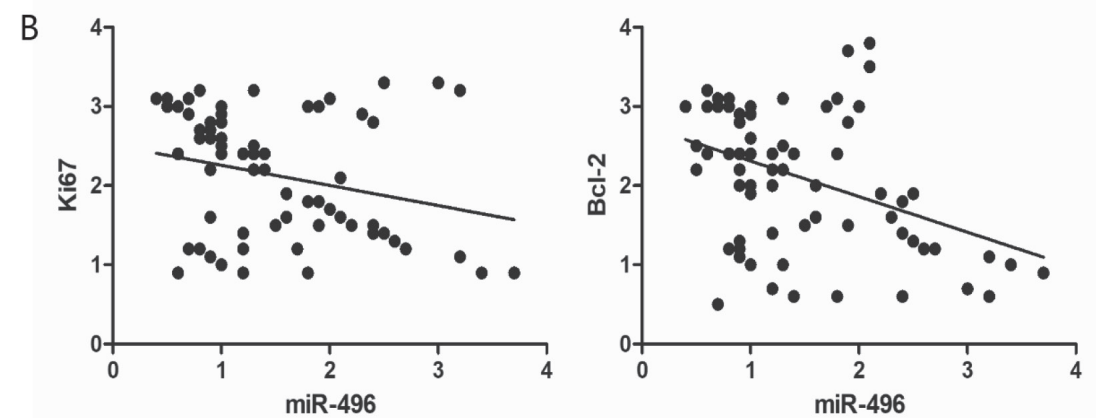

Figure 3. Correlation between miR-496 expression and Ki67and Bcl-2 protein levels.

(A) shows the results of western blot .... (B) shows correlation between miR-496 expression and Ki67 (left) and Bcl-2 (right) protein levels in the tumor tissue.

mote tumor progression (Wen et al., 2016). MiR-496 is a factor related to abnormal proliferation of tumor. Earlier studies have shown that miR-496 is expressed in normal human tissues and cells, while miR-496 is often decreased in neoplastic cells (Huat et al., 2016). The abnormal expression of miR-496 is regulated by associated factors. The down-regulation of methylated DNA binding protein 2 (MBD2) can significantly inhibit miR-496 in human breast cancer MCF-7 cell line and initiate cell proliferation, metastasis, and cell migration. It involves changes in a variety of molecular biological pathways (Alvarado et al., 2013). In addition, members of the translation initiation factor family may be downstream regulators of miR-496 (Qi et al., 2019). We found that eIF3h, which has the function of multidirectional regulation, is one of the miR-496 targets. According to several published studies, eIF3h activation can promote tumor growth, which is mainly due to the regulation of epithelial cell proliferation (Wang et al., 2018). However, after silencing of eIF3h,

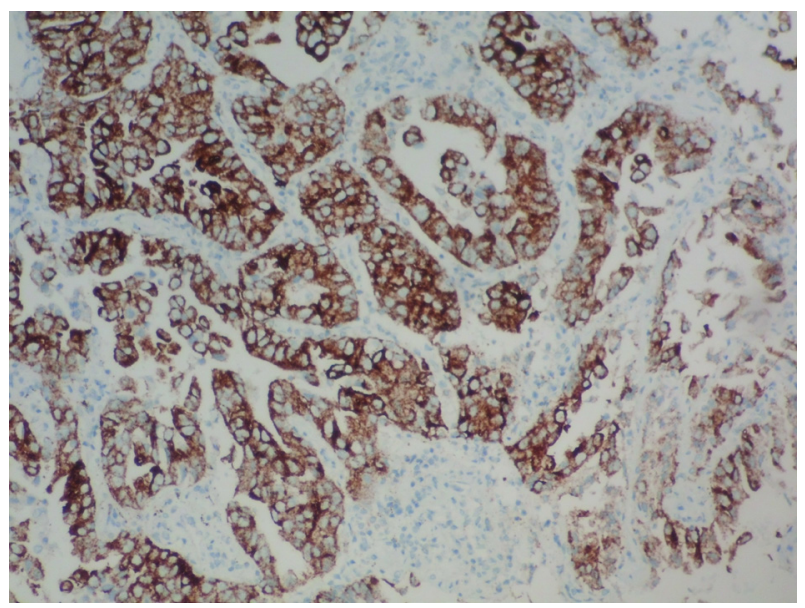

Figure 4. elF3h staining in lung adenocarcinoma. the proliferation activity of tumor cells decreased, the ability of cell division was poor, and the adhesion between cells decreased with the low ability of cell migration (Cappuzzo et al., 2009; Zhu et al., 2016).

This study is conducted with the use of pathological tissue of lung adenocarcinoma that was resected surgically. The accumulated results showed that the expression of miR-496 in lung adenocarcinoma was lower than that in normal lung tissue, which indicated that low expression of miR-496 was a crucial molecular factor in the formation of lung adenocarcinoma. In addition, we also found that miR-496 expression in tumor tissue from the patients with lymph node metastasis, pleural involvement and TNM stage III + IV was significantly lower than that of patients without lymph node metastasis, pleural involvement and TNM stage I + II. Wen and others (Wen et al., 2016) found that miR-496 was related to the occurrence and development of hepatocellular carcinoma. They found that when miR-496 inhibitor was added, the number of tu-

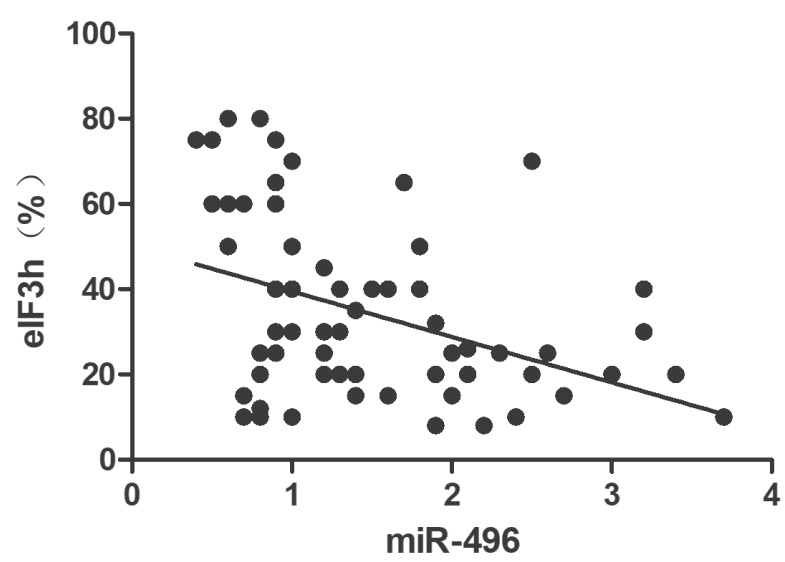

Figure 5. Correlation between miR-496 expression and the percentage of elF3h-positive cells in the tumor. 
mor cells in G0/G1 phase decreased and the number of tumor cells in G2/M phase increased. Zhou and others (Zhou et al., 2016) found that miR-496 had low expression in colorectal adenocarcinoma cells, and it inhibited the proliferation and metastasis of colorectal adenocarcinoma cells by regulating Wnt pathway activity. These reports are similar to the results of this study, suggesting that miR-496 is a tumor suppressor.

MiR-496 may regulate the proliferation of cells by regulating the expression of $\mathrm{Ki} 67$, and may have an effect on apoptosis by regulating the expression of Bcl-2 protein (Lu et al., 2018; Yao et al., 2019). In this study, we found that the expression of miR-496 had association with TNM stage. Considering that TNM is an important factor to evaluate the prognosis of tumor, we speculate that the expression of miR-496 may be related to prognosis of tumor. The results of survival analysis also confirmed our assumption that the low expression of miR-496 was accompanied with a poor prognosis. In addition, we found that the expression of miR-496 was not correlated with the degree of differentiation of lung adenocarcinoma, suggesting that miR-496 had no significant regulatory effect on stem cells and immature cells. In non-small cell lung cancer (NSCLC), miR-496 is reported to have similar effects. Research has shown that upregulated expression of miR-496 suppressed tumor growth via inactivating brain derived neurotrophic factor (BDNF)-mediated PI3K/Akt signaling pathway in clinical patients and in most of the NSCLC cell lines, including H1650, H292, H1944 and A549 (Bhardwaj et al., 2019).

Our study indicated that the expression of miR496 was negatively correlated with eIF3h. MiR-496 may be the upstream factor of eIF3h, which mediates tumor angiogenesis, cell adhesion and cell migration by regulating the translation process (Bhardwaj et al., 2019). Moreover, the research on the negative regulation of eIF3h in lung adenocarcinoma cells has always attracted attention. This study corroborates the negative regulatory effect of miR-496 on eIF3h. There are also studies describing a previously unknown negative regulation eIF3h by PDCD4, and eIF3h was also confirmed as an oncogenic factor in lung adenocarcinoma, where it acts via enhancing EMT signaling, which can be abrogated by PDCD4. This negative regulation mechanism will be further explored in future research.

In addition, according to Qi and others (Qi et al., 2019), eIF4E could be regulated by abnormal expression of miR-496 in human osteosarcoma, indicating that miR-496 may regulate many members of eIF family. Considering that there are only few studies on the regulation of downstream eukaryotic initiation factors by miR-496, more follow-up research is needed to further reveal these relationships. The prognostic value of miR-496 also needs to be verified by studies from multiple centers and with a larger number of samples and a longer follow-up time to further confirm the role of miR-496 as a prognostic factor.

\section{CONCLUSION}

In conclusion, the low expression of miR-496 is related to the progress of the lesion in lung adenocarcinoma. MiR-496 may have potential function in regulating eIF3h. Moreover, miR-496 may be an independent prognostic factor for lung adenocarcinoma.

\section{Acknowledgments}

None.

\section{Conflict of Interest}

The authors declare no conflict of interest.

\section{REFERENCES}

Alvarado S, Wyglinski J, Suderman M, Andrews SA, Szyf M (2013) Methylated DNA binding domain protein 2 (MBD2) coordinately silences gene expression through activation of the microRNA hsamir-496 promoter in breast cancer cell line. PLoS One 8: e74009. https://doi.org/10.1371/journal.pone.0074009

Bhardwaj U, Powell P, Goss DJ (2019) Eukaryotic initiation factor (eIF) 3 mediates Barley Yellow Dwarf Viral mRNA 3'-5' UTR interactions and $40 \mathrm{~S}$ ribosomal subunit binding to facilitate cap-independent translation. Nucleic Acids Res 47: 6225-6235. https://doi. org/10.1093/nar/gkz448

Cappuzzo F, Varella-Garcia M, Rossi E, Gajapathy S, Valente M, Drabkin H, Gemmill R (2009) MYC and EIF3H Coamplification significantly improve response and survival of non-small cell lung cancer patients (NSCLC) treated with gefitinib. J Thorac Oncol 4: 472-478. https://doi.org/10.1097/JTO.0b013e31819a5767

Choudhuri A, Maitra U, Evans T (2013) Translation initiation factor eIF3h targets specific transcripts to polysomes during embryogenesis. Proc Natl Acad Sci US A 110: 9818-9823. https://doi. org/10.1073/pnas.1302934110

Huat TJ, Khan AA, Abdullah JM, Idris FM, Jaafar H (2015) MicroRNA expression profile of neural progenitor-like cells derived from rat bone marrow mesenchymal stem cells under the influence of IGF-1, bFGF and EGF. Int J Mol Sci 16: 9693-9718. https://doi. org/10.3390/ijms16059693

Lu QC, Rui ZH, Guo ZL, Xie W, Shan S, Ren T (2018) LncRNADANCR contributes to lung adenocarcinoma progression by sponging miR-496 to modulate mTOR expression. J Cell Mol Med 22: 1527-1537. https://doi.org/10.1111/jcmm.13420

Meng W, Ye Z, Cui R, Perry J, Dedousi-Huebner V, Huebner A, Wang Y, Li B, Volinia S, Nakanishi H, Kim T, Suh SS, Ayers LW, Ross P, Croce CM, Chakravarti A, Jin VX, Lautenschlaeger T (2013) MicroRNA-31 Predicts the Presence of Lymph Node Metastases and Survival in Patients with Lung Adenocarcinoma. Clin Cancer Res 19: 5423-5433. https://doi.org/10.1158/1078-0432.CCR-13-0320

Mi J, Zou Y, Lin X, Lu J, Liu X, Zhao H, Ye X, Hu H, Jiang B, Han B, Shao C, Gong Y (2017) Dysregulation of the miR-194-CUL4B negative feedback loop drives tumorigenesis in non-small-cell lung carcinoma. Mol Oncol 11: 305-319. https://doi.org/10.1002/18780261.12038

Palmieri A, Pezzetti F, Spinelli G, Arlotti M, Avantaggiato A, Scarano A, Scapoli L, Zollino I, Carinci F (2008) PerioGlas ${ }^{\circledR}$ Regulates Osteoblast RNA Interfering. J Prosthodont 17: 522-526. https://doi. org/10.1111/j.1532-849X.2008.00331.x

Qi NN, Tian S, Li X, Wang FL, Liu B (2019) Up-regulation of microRNA-496 suppresses proliferation, invasion, migration and in vivo tumorigenicity of human osteosarcoma cells by targeting eIF4E. Biochimie 163: 1-11. https://doi.org/10.1016/j.biochi.2019.04.017

Rubie C, Kölsch K, Halajda B, Eichler H, Wagenpfeil S, Roemer K, Glanemann M (2016) microRNA-496 - A new, potentially agingrelevant regulator of mTOR. Cell Cycle 15: 1108-1116. https://doi. org/10.1080/15384101.2016.1158360

Savinainen KJ, Linja MJ, Saramäki OR, Tammela TL, Chang GT, Brinkmann AO, Visakorpi T (2004) Expression and copy number analysis of TRPS1, EIF3S3 and MYC genes in breast and prostate cancer. Br J Cancer 90: 1041-1046. https://doi.org/10.1038/ sj.bjc. 6601648

Wang X, Wang H, Zhao S, Sun P, Wen D, Liu T, Liu H, Yang Z, Ma Z (2018) Eukaryotic translation initiation factor EIF3H potentiates gastric carcinoma cell proliferation. Tissue Cell 53: 23-29. https:// doi.org/10.1016/j.tice.2018.05.006

Wen Y, Xu A, Jiang M (2016) Effect of miR-496 on cell cycle of HepG2 cell line. Journal of Jiangsu University of Science and Technology (Natural Science Edition) 30: 99-102. DOI: 10.3969/j.issn.16734807.2016.01.017

Wu Y, Zhang J, Hou S, Cheng Z, Yuan M (2017) Non-small cell lung cancer: miR-30d suppresses tumor invasion and migration by directly targeting NFIB. Biotechnol Lett 39: 1827-1834. https://doi. org/10.1007/s10529-017-2428-9

Yao X, Yao R, Yi J, Huang F (2019) Upregulation of miR-496 decreases cerebral ischemia/reperfusion injury by negatively regulating BCL2L14. Neurosci Lett 696: 197-205. https://doi.org/10.1016/j. neulet.2018.12.039

Zhang J, Du Y, Wu C, Ren X, Ti X, Shi J, Zhao F, Yin H (2010) Curcumin promotes apoptosis in human lung adenocarcinoma cells 
through miR-186* signaling pathway. Oncol Rep 24: 1217-1223. https://doi.org/10.3892/or_00000975

Zhang L, Pan X, Hershey JW (2007) Individual overexpression of five subunits of human translation initiation factor eIF3 promotes malignant transformation of immortal fibroblast cells. $J$ Biol Chem 282: 5790-5800. https://doi.org/10.1074/jbc.M606284200

Zhang L, Smit-McBride Z, Pan X, Rheinhardt J, Hershey JW (2008) An oncogenic role for the phosphorylated h-subunit of human translation initiation factor eIF3. J Biol Chem 7(10). https://doi. org/10.1074/jbc.M800956200
Zhou J, Zhong B, Liu L, Wang J (2016) miR-496 overexpression inhibits colon cancer cell growth and metastasis. Chinese J Pathophysiol 32: $1815-1823$

Zhu Q, Qiao GL, Zeng XC, Li Y, Yan JJ, Duan R, Du ZY (2016) Elevated expression of eukaryotic translation initiation factor $3 \mathrm{H}$ is associated with proliferation, invasion and tumorigenicity in human hepatocellular carcinoma. Oncotarget 7: 49888-49901. https://doi. org/10.18632/oncotarget.10222 